\title{
Factors that Contribute to Nursing and Medical Students' Perceptions of the Nurse-Physician Relationship
}

\author{
Kathryn G. Conover ${ }^{1}$, Michaela E. Behrens ${ }^{1}$, Emily N. Usenick ${ }^{1}$, Samuel P. Abraham ${ }^{1} \&$ Deborah R. Gillum ${ }^{1}$ \\ ${ }^{1}$ Bethel University School of Nursing, Mishawaka, Indiana \\ Correspondence: Samuel P. Abraham, Bethel University School of Nursing, 1001 Bethel Circle, Mishawaka, \\ Indiana, 46545 .
}

Received: May 27, 2019

Accepted: June 12, 2019

Online Published: June 21, 2019

doi:10.20849/ijsn.v4i3.597

URL: https://doi.org/10.20849/ijsn.v4i3.597

\begin{abstract}
Background: Biases and preconceptions regarding future working relationships have the potential to impact professional interactions. Purpose: The purpose of this qualitative study was to investigate the factors that contribute to nursing and medical students' perceptions of the nurse-physician relationship. Method: This was a qualitative, phenomenological, explorative study with a descriptive design. Eight nursing students and eight medical students were interviewed for this study and themes were carefully extracted from their responses. A series of open-ended questions were asked that were intended to elicit honest, thoughtful responses regarding how they view the other profession, their relationship, and thoughts on the quality of future professional collaboration. Social identity theory was used as the conceptual framework to guide this study. Themes were then extracted from the interviews. Results: The main themes found were in the areas of attitudes and behaviors. Nursing students had more positive than negative comments about interdisciplinary attitudes, but they had slightly more negative comments regarding interdisciplinary behaviors. Medical students commented far more on interdisciplinary behaviors than on attitudes, with both categories turning out more positive than negative. Specific subthemes included medical students' concern with sexism against female physicians and their high degree of concern over how interdisciplinary relationships affect the patient. Nursing students believed the relationship would be respectful depending on the unit. However, most of them expressed concern with how negative behaviors impact the patient. Conclusion: Learning standout themes from nursing and medical students' perceptions of their future working relationships can aid in finding barriers that can be addressed. Future research would be beneficial to discover themes on a larger scale.
\end{abstract}

Keywords: interprofessional perceptions, interdisciplinary collaboration, interprofessional education, nurse-physician relationships

\section{Introduction}

The Joint Commission on Accreditation of Healthcare Organizations (TJC, 2008) reports 70\% of sentinel events stem from inadequate interprofessional communication. This important aspect of communication can be altered by negative perceptions, creating a danger to patients (House \& Havens, 2017). Professional conflict can negatively impact patient safety by creating a barrier between coworkers who should communicate openly. It would be especially harmful if professional conflict began before either the nurse or physician graduated. There is a research gap in assessing the views of students independent of interprofessional education (IPE) experience. The purpose of this qualitative study was to investigate factors that contribute to nursing and medical students' perceptions of the nurse-physician relationship.

\subsection{Background}

Professional conflict between physicians and nurses (previously referred to as the doctor-nurse game and defined by feelings of hostility and disrespect) has been an issue for decades (Stein, 1967). In 1971, the American Nurses Association and American Medical Association supported the development of the National Joint Practice Commission, which birthed a series of successful interdisciplinary projects in response to antagonism concerns between nurses and physicians ("Guidelines for Establishing," 1974). Tang, Chan, Zhou, and Liaw (2013) conducted an extensive literature review and suggested that this form of interdisciplinary hostility continues to impact professional communication. 
The root of this hostility seems to be negative perceptions held by nurses and physicians about each other and assumptions of what the other group thinks of them (Collette et al., 2017; House \& Havens, 2017; Thomson, Outram, Gilligan, \& Levett-Jones, 2015; Vila, 2016). Many studies have investigated the prevalence and strength of these perceptions and how they impact patient safety. Others have made connections to students and how IPE impacts their sense of respect and collaboration (Kruegar, Ernstmeyer, \& Kirking, 2017). This study added to the limited knowledge of the perceptions held by students as well as their idea of the factors contributing to those perceptions.

\subsection{Research Questions}

The main research question was: What are factors that contribute to nursing and medical students' perceptions of the nurse-physician relationship? This question answered:

- What are nursing students' perceptions of the nurse-physician relationship?

- What are medical students' perceptions of the nurse-physician relationship?

- What do nursing students believe are the contributing factors relating to their perceptions of the nurse-physician relationship?

- What do medical students believe are the contributing factors relating to their perceptions of the nurse-physician relationship?

\section{Review of the Literature}

A thematic review of relevant literature is discussed below. Search procedure included the use of the Cumulative Index of Nursing and Applied Health Literature (CINAHL), Medline Full-Text, Nursing@Ovid, and EBSCOhost databases. Keywords used to find these articles were interprofessional perceptions, interdisciplinary collaboration, and interprofessional education. Articles written from 2015 to 2018 were reviewed along with seminal research and relevant studies found in the reference page of recent studies.

\subsection{Interprofessional Perceptions}

This section aims to address what the researchers found in the academic literature regarding what nurses and physicians think of each other in general. The main themes in interprofessional perceptions are in the areas of respect and role appreciation. Understanding these perceptions will highlight areas of needed intervention (House \& Havens, 2017).

Regarding respect, nurses generally carried more preconceptions about physicians than the other way around. No significant negative comments by physicians were found regarding nurses. Physicians tended to report positive views of nurses (Collette et al., 2017; Lancaster, Kolakowsky-Hayner, Kovacich \& Greer-Williams, 2015). However, nurses often reported negative characteristics of physicians, most frequently that they were arrogant, disrespectful, and authoritarian (Collette et al., 2017; Lancaster et al., 2015). Thomson et al. (2015) noted, "perceptions of others' beliefs and attitudes were often in conflict with the perceptions reported by these other professionals" (p. 638).

Brown, Hochstetler, Rode, Abraham, and Gillum (2018) discovered most of their study participants, all of whom were newly graduated nurses, expressed feelings of intimidation when working with physicians. Feelings of intimidation began before they entered the nursing field, based partly on what other people had told them. The study results indicated that their perception was not substantiated with their actual relationship experience with the physicians during their first year of practice (Brown et al., 2018). Actual experience was positive and not intimidating as perceived.

Role appreciation also seems to be included in the discrepancy between the healthcare team members. Throughout the literature review, nurses expressed a desire to be appreciated by physicians (Collette et al., 2017; House \& Havens, 2017; Lancaster et al., 2015; Thomson et al., 2015). Lancaster et al. asked nurses how they thought physicians viewed their role, and they responded that physicians considered themselves superior to nurses. Interestingly, the interviewed physicians reported appreciation for the role of the nurse, with many calling them "an integral part of patient care" (p. 279).

\subsection{Nurse-Physician Collaboration}

It is important to assess how nurses and physicians view collaboration. Instead of considering themselves as all one team, new graduates tend to identify strongly as part of their own group of either nurses or physicians (Thomson et al., 2015). This correlates with Tajfel's (1982) social identity theory in its assumption that individuals automatically put themselves into an in-group to increase self-esteem. Schaik, O’Sullivan, Eva, Irby, 
and Regehr (2016) discovered that nurses and physicians were more likely to guess that helpful feedback came from members of their own profession.

Collaboration may be strongly influenced by the department in which one works. Departments with increased interactions between nurses and physicians, such as emergency departments, have higher perceptions of effective collaboration than departments with less interaction within the hospital setting (Collette et al., 2017; House \& Havens, 2017; Lancaster et al., 2015).

\subsection{Interprofessional Education}

Scheduled interprofessional education has shown to improve working relationships. Specifically, IPE improves interdisciplinary perceptions and collaboration (Collette et al., 2017; Krueger, Ernstmeyer, \& Kirking, 2017). Evaluation of IPE is also helpful because it is the context of the studies that address perceptions of students. House and Havens (2017) suggested increased IPE, including combined classes, for medical and nursing students. Learning to work together and respect each other before graduating may significantly decrease negative perceptions and increase knowledge of necessary steps for effective collaboration. Collaborative learning could then easily go beyond the classroom into the professional environment (Lancaster et al., 2015).

\subsection{Summary of the Literature Review}

Overall, nurses tended to have an increased negative perception of physicians than physicians had of nurses. Views on the quality of collaboration vary, but departments that naturally have a high degree of mutual decision-making between physicians and nurses were found to have higher satisfaction with collaboration than departments that were more segmented. IPE has shown to improve the quality of interprofessional perceptions and collaborative values. The review of the literature highlighted the need for interventions to improve interprofessional working relationships between physicians and nurses and provided information on student perceptions within the context of IPE experiences.

\subsection{Theoretical Framework}

Social identity theory (SIT), created by Tajfel (1982), was the theoretical base for this study. SIT offered a unique perspective on how nurses and physicians relate to each other in the workplace, particularly regarding the presence of bias against the other profession. This theory claims a need for self-esteem drives people to categorize themselves with others, thereby creating an 'in-group' of similar people. To increase self-esteem, members of the 'in-group' are predicted to villainize the 'out-group.' Villainizing the out-group fosters a sense of unity within the 'in-group' and increases the individual's self-esteem and sense of belonging (Tajfel, 1982).

Physicians and nurses, while technically belonging to the same healthcare team, are in disciplines with different roles. These roles often conflict with priorities and time management necessities, lending itself well to antagonism and resentment. However, this resentment may start before the professional's graduate because it could be passed down from working members of the healthcare teams to the students, so they may learn to identify themselves as an 'in-group' very early. This study explored how students categorized themselves into an 'in-group,' how they viewed the 'out-group,' and what factors influenced their perceptions. This information can be used to impact divisional mindsets before graduation for the betterment of the nurse-physician relationship.

\subsection{Definition of Terms}

\subsubsection{Operational Definitions}

Perceptions of students are defined in this study as what students believe will be true in the future, once they have graduated. This study examined perceptions of the nurse-physician relationship, which means the way that nurses and physicians will interact professionally after graduation. Collaboration is when nurses and physicians work together positively and contribute to patient care together with effective communication. Nursing students in this study are college students 18 years of age or older. Medical students are students in medical programs within the northern Indiana region who are 18 years of age or older. Interprofessional education is the scheduled, academic teaching of roles and communication skills in the classroom (pre-graduation) setting and the clinical (post-graduation) setting.

\subsubsection{Conceptual Definitions}

Group identification is composed of a cognitive understanding of membership in a group, an evaluative approval of the group's values, and an emotional investment in the group (Tajfel, 1982). Social identity describes the aspect of self-esteem that comes from belonging to an 'in-group.' To increase self-esteem, members of the 'in-group' negatively stereotype people in the 'out-group.' Stereotyping is exaggerating characteristics and applying them to all members of a group (Tajfel, 1982). 


\section{Methodology}

\subsection{Design}

This was a qualitative, phenomenological, exploratory study. Convenience sampling was used. Access to participants was through personal acquaintances, professional connections, and email invitations. The interviews lasted between 45-60 minutes and were conducted in a quiet place on campus. Medical students were interviewed on campus or a quiet and safe place suitable to the participant. Approval from the Institutional Review Board was obtained before conducting this research. The researchers for this study were certified through training regarding ethical research procedures through the National Institute of Health.

\subsection{Setting and Sample}

Eight nursing students and eight medical students were interviewed for a total of 16 participants. The sample consisted of male and female participants age 18 years and older. The college, the site from which the nursing student sample was obtained, is an educational institution in northern Indiana. The participating nursing students were in the Bachelor of Science in Nursing program in their sophomore or junior year. Medical students, of various years in their program, were acquaintances from the northern Indiana region. Snowball sampling was used for the medical students; where one research participant will help to recruit other participants for this study.

\subsection{Plan for Data Collection and Analysis}

Data was collected and analyzed using the Colaizzi's process (Speziale \& Carpenter, 2007). Semi-structured, face-to-face interviews were conducted using a pre-prepared interview guide. Participants were encouraged to talk freely and to tell stories using their own words. Each interview lasted from 45 minutes to one-hour, and the researchers conducted all interviews. At the end of each interview, the researchers reminded participants about their need for a second contact with them via telephone calls or face-to-face to clarify the first interview themes, to ensure accuracy, and to provide an additional chance to share thoughts and perceptions. Interviews were recorded by the researchers on their phones, which were locked with a passcode to maintain confidentiality. The researchers took notes during the interview and then following the interview transcribed each interview verbatim into a Word document that was kept locked and secured for confidentiality. The phone recordings were deleted after the data was transcribed.

Colaizzi presents the following steps for phenomenological data analysis (Speziale \& Carpenter, 2007):

$\checkmark$ Each transcript was read and re-read to obtain a general sense about the whole content.

$\checkmark \quad$ For each transcript, significant statements that pertain to the phenomena under study were extracted. These statements were recorded on a separate sheet noting their pages and line numbers.

$\checkmark \quad$ Meanings were formulated from these significant statements.

$\checkmark \quad$ The formulated meanings were sorted into categories, clusters of themes, and themes

$\checkmark$ The findings of the study were integrated into an exhaustive description of the

$\checkmark$ phenomena under study.

$\checkmark$ The fundamental structure of the phenomena was described.

$\checkmark$ Finally, validation of the findings was sought from the research participants to compare the researcher's descriptive results with their experiences.

\subsection{Interview}

The research questions and question prompts were developed based on a thorough review of the literature. The open-ended questions were intended to elicit detailed, thoughtful responses from the interviewee. The interview questions and outline were sent to two peers and two professors to be reviewed and to establish face-validity. Schmidt and Brown (2019) defined face-validity as the process of colleagues examining an interview instrument to determine if it measures what it is designed to measure. Upon analysis and feedback from peers, revisions were made to the interview statements as necessary.

\subsection{Informed Consent and Confidentiality}

Each participant was required to sign an informed consent. Included in the consent form is the assurance that the participant's name, personal information, or any other identifiers would not be connected to their opinions and responses of the interview. This confidentiality assured participants that their responses will have no personal consequences. The research data was stored and locked in a cabinet at the school of nursing. Only the nursing administrators and research coordinators had access to these records. 


\section{Results/Data Analysis}

Of the 16 students interviewed, eight were medical students and eight were nursing students. Seven of the participants were male and nine were female. Of the seven males interviewed, three were nursing students and four were medical students. Of the nine females interviewed, five were nursing students and four were medical students. Eleven of 16 participants were ethnically white or Caucasian. Eleven of the participants were in the 21-23 age range category, three were 24-26 years old, and two were 27 years old or above. 9 of the participants were in their junior/third-year of schooling respectively, four were first-years, one sophomore/second-year, and two senior/fourth-years. See Table 1 for demographic information.

\subsection{Demographics}

Table 1. Descriptive statistics for participant demographics and background

\begin{tabular}{lll}
\hline Variable & Frequency (f) & Percentage (\%) \\
\hline Gender & 7 & \\
\hline Male & 9 & $54 \%$ \\
Female & & $56 \%$ \\
\hline Ethnicity & 11 & $69 \%$ \\
\hline White/Caucasian & 1 & $6 \%$ \\
Hispanic or Latino & 2 & $12.5 \%$ \\
Black or African American & 2 & $12.5 \%$ \\
Asian & & \\
\hline Age (years) & 11 & $69 \%$ \\
\hline 21-23 & 3 & $19 \%$ \\
$\mathbf{2 4 - 2 6}$ & 2 & $12 \%$ \\
27 or above & & \\
\hline Type of Student & 8 & $50 \%$ \\
\hline Nursing Student & 8 & $50 \%$ \\
Medical Student & & \\
\hline Class & 4 & $25 \%$ \\
\hline Freshman/First-year & 1 & $6.25 \%$ \\
Sophomore/Second-year & 9 & $12.5 \%$ \\
Junior/Third-year & 2 & \\
Senior/Fourth-year & & \\
\hline & & \\
\hline 6 & & \\
\hline
\end{tabular}

Note: $\mathrm{N}=16$

\subsection{Themes}

Themes were derived from the 16 interview results and fell into four categories. The themes were further separated into nursing student interviews and medical student interviews resulting in two themes for each. The themes were as follows for nursing students: (1) attitudes, (2) behaviors. Themes were as follows for medical students: (3) attitudes, and (4) behaviors. See Figure 1 and Figure 2. Student participants were labeled as P1 to P16. 


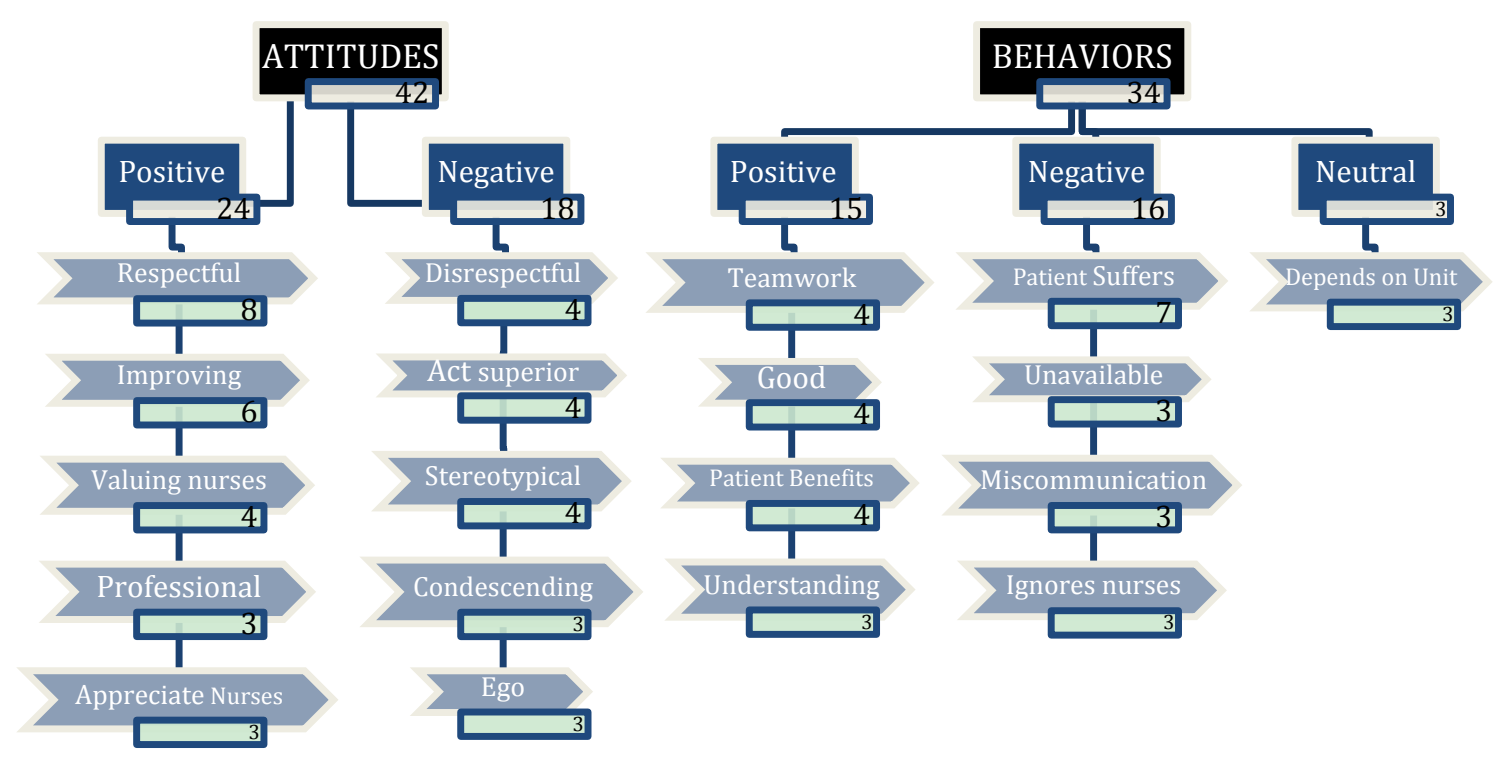

Figure 1. Common themes found in 8 nursing student research interviews

\subsubsection{Nursing Student Theme 1: Attitudes}

One of the first trends to emerge in our responses was the inverse emphasis that our two groups had on the major themes. For nursing students, themes regarding attitudes that physicians and nurses hold about each other were talked about more frequently than themes about behaviors towards each other. Conversely, medical students emphasized themes of behavior more frequently than themes about attitudes. This section will offer more detail about positive and negative subthemes from the perspective of our nursing student interviewees.

\subsubsection{Positive}

Most of the subthemes from nursing students regarding assumptions were positive. In general, the students were cautiously optimistic, preceding many of their statements with the phrase, "well hopefully..." All eight nursing students indicated they perceived their future working relationship would be respectful. Participant 6 represented many of the others' thoughts when they explained, "More often than not the doctors are super friendly and talking to the nurses all the time. I feel like when I get on the floor it's not going to be so bad."

Similar expectations were shared in other subthemes, including the belief that the nurse-physician relationship would be professional and one where physicians valued nurses for their contributions and appreciated their role. This general assumption was held by participant 1 who stated, "If I do have a concern, I think they will respond and take my concerns to heart and not just [ignore me]."

One of the more unexpected subthemes was how most of the nursing students mentioned that attitudes within the nurse-physician relationship are getting better with time. One of the voices for this majority perception, participant 3, contributed an added level of insight when they said, "I think it will get better... as education for nursing improves [and with] learning how to appreciate the doctors. Or med school teaching how to appreciate their nurses. I think it will get better because hospitals are focusing on collaborative mindsets and being a team instead of being just an individual."

\subsubsection{Negative}

Even though all eight nursing students indicated they believed their future working relationship with physicians would be respectful, the major negative subthemes included the expectation that nurses and physicians are disrespectful of each other. For the most part, students that indicated both "respectful" and "disrespectful" conceded their perception of the default setting is respectful while the minority of the time having disrespectful perceptions of the other.

Participant 12 added, "I think a lack of respect is evident from both sides in a lot of cases. Like I said earlier, 
some doctors just don't respect nurses I think." The other participants that expected some degree of disrespect agreed that the disrespect they think would occur in both directions. However, they seemed to attribute physicians' disrespect as coming from their job position while mostly claiming disrespect from nurses comes from their specific personalities.

Other more common negative responses included the belief that physicians think of themselves as superior to nurses. Participant 6 stated, "I feel like not all doctors are going to be as respectful because the stereotype is that nurses are [just] medication pushers and footstools for the doctors."

\subsubsection{Nursing Student Theme 2: Behaviors}

The nursing student participants had less to say about their perceptions of future behaviors between physicians and nurses than they said about perceived attitudes. This difference may indicate that nursing students have a greater concern for how the physicians think about them than how they interact. Despite being mentioned less, behaviors were the only thematic category for either nurses or physicians that had a larger negative leaning (by only one point) than positive.

\subsubsection{Positive}

The nursing students identified positive behavioral expectations that were general in nature, with an overall emphasis on thinking it will be good. Half of our participants specifically listed positive perceptions of future teamwork. "I expect that [the relationship is] going to be collaborative and the doctors will work with me and help me," explained participant 3. That same nursing student further developed that thought by adding "I think what I have experienced will be how I experience things outside nursing school in the nurse role. I have had good experiences and I think that as long as you treat them with respect, and you value their input and accept constructive criticism [it will be good]."

Half of the total participants also mentioned how positive, collaborative behaviors benefit the patients. Participant 6 explained, "I think that communication and working together and collaborating together shows [the patient] that the nurse is not just doing what the doctor says but [shows] they have a mind of their own." Demonstrating nursing independence to patients was the more common positive benefit mentioned. For our participants, they expect future patients to listen to them and trust their care more fully when the nurse and physician work collaboratively.

\subsubsection{Negative}

The most commonly held negative perception of their future working relationship with doctors was the recognition that it would be bad for the patients. Some mentioned under positive behavior perceptions that a good working relationship would benefit the patient, but the converse was more commonly mentioned. Participant 6 explained, "I think the communication between nurses and doctors in front of the patient is really what has, in the past, affected how patients see nurses because the doctor comes in, sees the patient, tells the nurse [what to do], and then the doctor leaves and the nurse does whatever he says."

This includes the added factor of negative working relationships being played out in front of the patient rather than simply behind the scenes. Participant 3 added similar beliefs, "Patients catch on to a lot of things faster than we think they do. Usually nurses come in alongside the doctors and if they don't feel like [it is] collaborative, they can sense a negative work environment and they won't be sure whose judgment to trust. Most people will want to trust the doctor more than the nurse... if it looks like there is competition it might have an effect on how well they take information or education."

These expectations were shared by the majority of nursing students. Not only do they believe that positive behaviors will be a benefit to patients, but also negative behaviors can be quite detrimental to patient outcomes. Participant 11 emphasized specific consequences of a negative work environment "They both are caring for the patient and if they don't collaborate together that is how miscommunication happens, this is how medication errors, or any type of errors happen with the patient. Especially if there is no communication between them. So, if you are not seeing the doctor, as a nurse, and giving them updates, then I think that can really impact the patient in a bad way." This goes along with when The Joint Commission on Accreditation of Healthcare Organizations (TJC, 2008) reported $70 \%$ of sentinel events stem from inadequate interprofessional communication. Nursing students seem to be aware of this important link.

Other perceptions of negative behaviors included the belief that unavailability of the physicians is an expected negative aspect of the nurse-physician relationship. Participant 3 echoed the others' opinions on this issue when they added, "As a nurse you might feel frustrated when the doctor isn't available, but you feel like the patient needs something." This is yet another way that negative behaviors can affect the patient, but with more 
specificity to the cause.

\subsubsection{Neutral}

Nursing students' perceptions of behaviors is the only theme that includes a neutral subtheme component. Three participants stated that the behaviors between nurses and physicians largely depends on the work setting. Participant 12 stated, "I think it depends on the floor you are on... Most of the time, I think nurses and doctors are respectful of each other." The three participants who included this observation indicated that areas with high amounts of collaboration, like the Emergency Department, would probably have more positive behaviors. This theme did not emerge from the medical students as a whole group however, one medical student participant mentioned it. Participant 16, a medical student, stated, "I feel like that is a challenging question because it is dependent on every location." He went on to explain that "so commonly a lot of the comments I get from anyone in nursing is 'oh man, don't be an A-hole, all surgeons are A-holes, especially to nurses." Indicating that nursing students/nurses have an altered reaction to the nurse-physician relationship dependent on the location of practice.

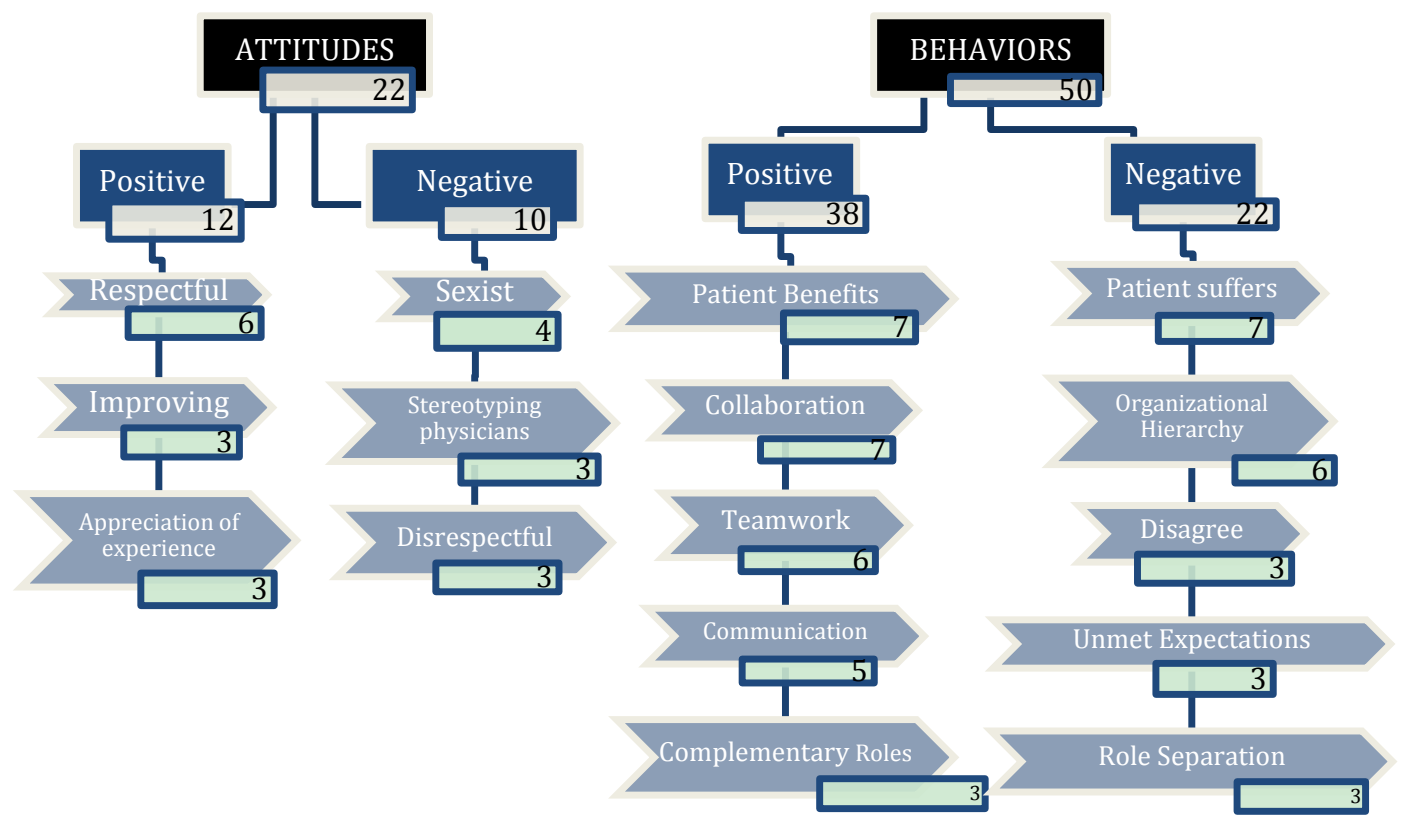

Figure 2. Common themes found in 8 medical student research interviews

\subsubsection{Medical Student Theme 1: Attitudes}

Nursing student participants mentioned how they perceived future attitudes more than future behaviors. Conversely, medical students mentioned perceived future behaviors far more often than they mentioned attitudes. While they mentioned expectations of positive attitudes slightly more often than negative ones, they did not heavily focus on attitudes in general.

\subsubsection{Positive.}

Overall, medical students were very general when speaking of their perception of expected attitudes of physicians and nurses, with the majority saying they expect reciprocating attitudes to be respectful. Three participants mentioned how there used to be more negative attitudes towards each other, but that it has been improving steadily over the years. Participant 2 mentioned, "I know there was an old-fashioned power dynamic, and I would say that is not what I see most of the time... I hear from older nurses that it is better than what it used to be." Similarly, two of these participants mentioned the difference they see in the upcoming generation of doctors and nurses. A great example of this is from participant 4. "I think it is getting a lot better. It seems like younger nurses are a lot less likely to have [negative] sentiments... it seems like younger physicians are more respectful of nurses and there seems to be a shift in hospital culture."

An improving mindset is a great aspect of the nurse-physician relationship to notice. In addition to seeing attitudes improving among professionals, some medical students had largely positive things to say about their attitude towards nurses in general. Participant 4 included, "The nursing profession right now knows way more 
than we [do]. You guys have a lot of insight that you can provide especially to more novice doctors." This shows an appreciation for the level of knowledge that nurses have. When asked about their expectations for the nurse-physician relationship, participant 5 even included, "I automatically think [the nurses know] what is going on when I don't."

\subsubsection{Negative}

One of the more interesting differences between themes of nursing and medical students was the different in their perceptions of negative attitudes. Four medical student participants, all of whom are female, expect nurses to be sexist against female physicians. Participant 5 stated it best when she said, "I know with female med students and doctors it is harder to establish the [nurse-physician] relationship because there is less respect." Their expectation of sexism applied even to female nurses, who they believe still respect female physicians less than their male colleagues. This was not a theme with our female nursing students, who anticipated any disrespect to be from role differences instead of from gender.

Another expressed perception of negative attitudes between nurses and physicians was the belief that nurses often stereotype physicians as similarly bad. Participant 16 added, "I think sometimes nurses can view physicians as mean bosses who just expect their orders to be blindly followed without any sort of feedback." Interestingly, one of the nursing students' themes for negative attitudes was centered on the typical physician stereotype, which seems to partly confirm the medical students' fears.

\subsubsection{Medical Student Theme 2: Behaviors}

In contrast to the few times perceived attitudes were mentioned, the perceived behaviors of the nurse-physician relationship were a focus for our medical student participants. Most of the comments focused on behaviors were positive, presenting a hopeful outlook for their future working relationship with nurses. Overall, most of these responses circled back to how the patient is affected.

\subsubsection{Positive}

All but one of our medical student participants talked about nurse-physician behaviors within the context of how it affected the patient. This is similar to our responses from nursing students, but there is more of majority with medical students and is one of the top subthemes for both positive and negative behavioral expectations. The medical students we interviewed were clearly keeping their future patients in the forefront of their mind. Participant 8 exemplified these concerns when they explained, "I think it has a huge impact on the patient how well nurses and physician work together. That can determine the pace at which patients can see their physicians and it's important for nurses to be able to communicate their thoughts and ideas about the patient to the physician, so they can get a proper plan in place."

The other subthemes were similar in nature, with teamwork, collaboration, and having complementary roles being frequently mentioned. Participant 9 spoke to the concept of complementary roles when they added, "It is basically two separate roles with the same end game. The role of the physician is making overall decisions and the nurse is generally at the bedside every day and they have as much of a right to manage the patient as well."

\subsubsection{Negative}

Similar to what was found with medical students' perceptions of future behaviors between nurses and physicians, the effect on the patient was the most common subtheme when discussing expected negative behaviors. Participant 2 stated, "I think sometimes [there] can be conflict in front of the patient. I have seen that multiple times where we disagree on what to do." This expectation of conflict sometimes occurring in view of the patient was also shared by the nursing students. Emphasis on the patient was put succinctly by participant 5, "Lack of communication will affect patient care." Participant 15 even threw an analogy into the mix to make their point, "I am a big sports analogy guy so if the line and the quarterback aren't communicating well, neither one of them are going to be successful, or the wide receiver or whatever. So, if the nurses and doctors, if one person is doing a great job but they aren't communicating well with the other people then that is going to result in a poor outcome for the patient."

One of the other subthemes was the expectation of unmet expectations from both sides of the professional isle. Participant 2 added, "I think there are a lot of times where you see that the order didn't get done but there might be other things going on with a different patient or a different emergency that you didn't see. And then on the other side nurses seeing physicians not doing things they should have done but they didn't see what the physicians [were] doing during that time."

These unmet expectations can be exacerbated, from the medical students' perspective, by the structure of 
healthcare. A few participants mentioned that the very nature of the role difference between physicians and nurses can make it seem like one is more important than the other. While this was classified as a negative perception of behavior, every participant that talked about this mentioned that the hierarchy in itself was negative, and they wished it didn't lead to feelings of superiority. They did not appreciate this aspect of healthcare. Participant 4 added, "The hospital system is set up in a hierarchical structure where traditionally you would think, 'oh the nurse needs to... follow the doc's orders' which I tend to not agree with very much."

Participant 16 had a poignant way of addressing the possible origin, and consequence, of continued negative perceptions, attitudes, and behaviors, "I think that it is really hard to build a good relationship from the ground, from a foundation. That foundation is built upon 'treat me well or I am going to make your life miserable' and that is from both sides. Doctors [are] saying 'the nurses better treat me well or I am going to be a jerk to them.' And nurses [are] saying 'these doctors or med students better treat me well or else I am going to make them absolutely miserable.' I think that is probably from years and years of negativity. They have kind of come to the point of 'oh we kind of have to be like this or we are going to be walked all over' from either side. I just don't think that is the place to start; that starts it off on the wrong foot from the very beginning."

\subsection{Contributing Factors}

From the 16 student interviews there were 8 contributing factors mentioned that influence their perception of the nurse-physician relationship. Contributing factors were as followed: (1) school, (2) work, (3) clinical, (4) friends/family, (5) nursing students, (6) nurses, (7) TV shows, and (8) doctors. See Figure 3 for comparison of contributing factors between nursing and medical students.

\subsubsection{Contributing Factor 1}

The first factor that contributed to how nursing students and medical students viewed the nurse- physician relationship is how their education and their professors influenced their perception of said relationship. Participant 1 explained, "I think what our [professors] have said [has contributed]. They have talked about how nurses are ignored, they're not taken very seriously... a recurrent cycle of saying things like that." Participant 12 also had experiences similarly to Participant one's experiences. When asked about they said, "I think sometimes our professors can paint it either really positively or really negatively. So, sometimes they will be very blunt with us when we ask a question or talk about our patient during clinical just because we aren't being specific enough or we didn't look up this lab to know this condition or whatever the case may be and just making it seem like if you call the doctor like that you are going to be hung up on and you are not going to get the answers you need, or the medication your patient needs because you don't know how to communicate to the doctor."

Participant 12 also added, "I think my perception has been greatly influenced my professors, my peers, and the nurses on the floors so professors can either talk great about doctors they have worked with or they can talk super negatively and say that they were super intimidating and don't care what nurses have to say so that automatically makes you think that all doctors are like that, like all doctors are scary to call or whatever. But then in clinical you see doctors and nurses working together and talking about getting together on the weekend and you see how they are still friends outside of work and their relationship is more than just as coworkers."

\subsubsection{Contributing Factor 2}

The second factor that contributed to how nursing students and medical students viewed the nurse- physician relationship is how their work experiences outside of clinical have impacted their views on the nurse- physician relationship. When asked about how work has influenced their perception of the nurse- physician relationship Participant 3 said, "I work a lot of times on a pediatric floor and the physicians there are so amazing. They give me the hope for other physicians. They are very warm towards the nurses and they trust the nurses' judgments and input. They want the nurses with them when they talk to the patients because they know that these nurses know the patients more than they do... It's just a very respectful environment."

Participant 13 talked about their work experiences and how it influenced their perceptions, "I mean I have seen it at work, as I gave the example of the doctor who complained because he had to walk to the other side of the hall - and it just puts those nurses in a bad mood and that is all they can think about and I can tell it made them really upset that something as small as that happened so if there is a bad relationship that is going on with the doctors it can really affect the nurses attitude and that greatly impacts the nurse's ability to care take for patients." Lastly, Participant 14 talks about how work has not affected her perception, "So my work experience, and seeing what I have seen, for the most part I have not seen anything that would change my view or my mind on them."

\subsubsection{Contributing Factor 3}

The third factor that contributed to how nursing students and medical students viewed the nurse physician 
relationship is how their clinical experiences have shaped their perception of the nurse- physician relationship. Participant 2 had two separate experiences in clinical. The first was the following: "One thing I'll never forget I was in the cardiac ICU and one of the cardiologists told me the most important thing is to make sure the nurse is in the room when you're seeing the patient because then you can answer all of their questions." The second was a negative experience that they faced during clinical that shaped their perception as described here, "One of the worst experiences that I had was when I felt like I didn't know what I was doing but I needed to help me with a patient who needed a bed pan... The nurse totally blew me off three times and I had to figure it out by myself. I don't think that was the best situation for the patient."

Participant 16 said the following about a clinical experience, "From what I see, face-to-face the doctors pretty much treat every nurse the same to their face and then will either just tell me the good or the bad things afterwards, kind of behind their back. So to their face it is certainly positive, and they get along well and they try to be really nice to them because almost all doctors make a point to tell me: "the nurses will make or break you, you have to be nice to them, they can make your job hell if they choose to, and they can make it really miserable." Participant 14 describes a pleasant clinical interaction; "I did have a very good experience with the doctor I had for my clinical when we walked around to each individual patient. Very open, very talkative, very approachable. So, I would say overall I have had good impressions of doctors."

\subsubsection{Contributing Factor 4}

The fourth factor that contributed to how nursing students and medical students viewed the nurse-physician relationship was stories they have heard or experiences they have had with family and friends in a health care setting. Participant 9 talked about a personal experience he witnessed and how it impacted his outlook on the relationship between nurses and physicians. "My twin sister she had cancer and went through the whole bone marrow transplant and all of the cancer treatment. The nursing experience I had there has had the greatest impact on how I view nurses because they were so involved all the time, so invested."

Participant 15 talks about a situation within their family, "I have several nurses in my family, and also have a lot of doctors too so I kind of have overture of seeing both sides and I also have only talked to the nurses that work with the doctors I have spent time with and I have had a really good picture of the both sides, of the physicians side, and the nurses side and thoughts and views and challenges they go through." Participant 16 talks about things they have heard through family conversations, "I hear rants from my friends that are nurses about stupid doctors who did this or that, but they also rant about stupid nurses who did this or that." Lastly, Participant 11 said the following; "I receive most of my stories about the nurse-physician relationship from my friends who work in hospitals as techs. They will tell you everything about the doctors, and how the nurses and their relationships are."

\subsubsection{Contributing Factor 5}

The fifth factor that contributed to how nursing students and medical students viewed the nurse- physician relationship was nursing students that they had interacted with. Whether it was in clinical or work or in the classroom. Both nursing students and medical students were impacted by stories and experiences with other nursing students. Participant 5 describes an experience they had, "I was at [an] IPE thing [with] 6-10 nursing students and they all looked down on the med students [and said], 'unlike some people, I look at the whole person.' Or, 'I wanted to be a nurse because I actually care about people' and they looked right at the med students... That automatically put in my brain that deep down all nurses resent physicians... I was shocked at the level of dislike they had for medical students."

Participant 5 said this about working with nursing students, "I picture [the communication] being friendly and hopefully with a high respect level. I'm going to be respecting them because they're running the show, but I hope it's reciprocated. I don't anticipate the respect being reciprocated in my head. I don't know why... probably because [of] my interactions with nursing students... there is a lot of animosity and feeling like they have to prove themselves."

\subsubsection{Contributing Factor 6}

The sixth factor that contributed to how the nursing students and medical students viewed the nurse- physician relationship was interacting with other nurses during clinical or work. Participant 16 describes how they view this interaction in the following statement, "To be honest, I probably hear the most about the nurse-doctor relationship from nursing students or nurses. It seems to be something they really like to talk about; especially when they find out I am a medical student." Participant 7 describes working with nurses and how they have helped positively shape her perception. "Working with nurses that have been in the field a little bit longer and 
hearing their perceptions which are either really good or really poor. So, perceptions from meeting nurses who have been in the field longer. I think I have more positive perception of doctors."

\subsubsection{Contributing Factor 7}

The seventh, and last factor that contributed to how the nursing students and medical students viewed the nursephysician relationship was watching medical shows on television. Before having experiences in surgery, Participant 13 talks about how television shaped her views of the nurse- physician relationship. "I think some of the biggest contributing factors, I think a lot of it is work, for me personally. Just because I see so much of it at work. However, before I worked as a pre-op surgical tech, I would have based it off TV shows or movies." Participant 3 describes how watching medical shows changed her perception of the relationship, "My negative perceptions, this is going to sound stupid, come from medical shows... a lot of them focus on the doctors and you never see the nurses. If there is a nurse, she is usually being berated by the doctor when she did something stupid."

\subsubsection{Contributing Factor 8}

The eighth contributing factor that contributed to perceptions of the nurse-physician relationship was derived from working with or talking to doctors. This factor was only mentioned by medical students. Participant 15 stated, "And then from the doctors perspective there is always the stories of the nurse calling him in middle of the night about something the nurse doesn't need to call the doctor about and then not thinking what they are saying on the call..." Participant 16 also explains his experience in working with physicians, "I have heard a lot of doctors who are like 'man, this nurse calls me for everything and it is really annoying or I will put in orders for something and she questions me all the time because she thinks she knows what's right' or something like that. So, I think most of the relationship, from what I see, face-to-face the doctors pretty much treat every nurse the same to their face and then will either just tell me the good or the bad things afterwards, kind of behind their back."

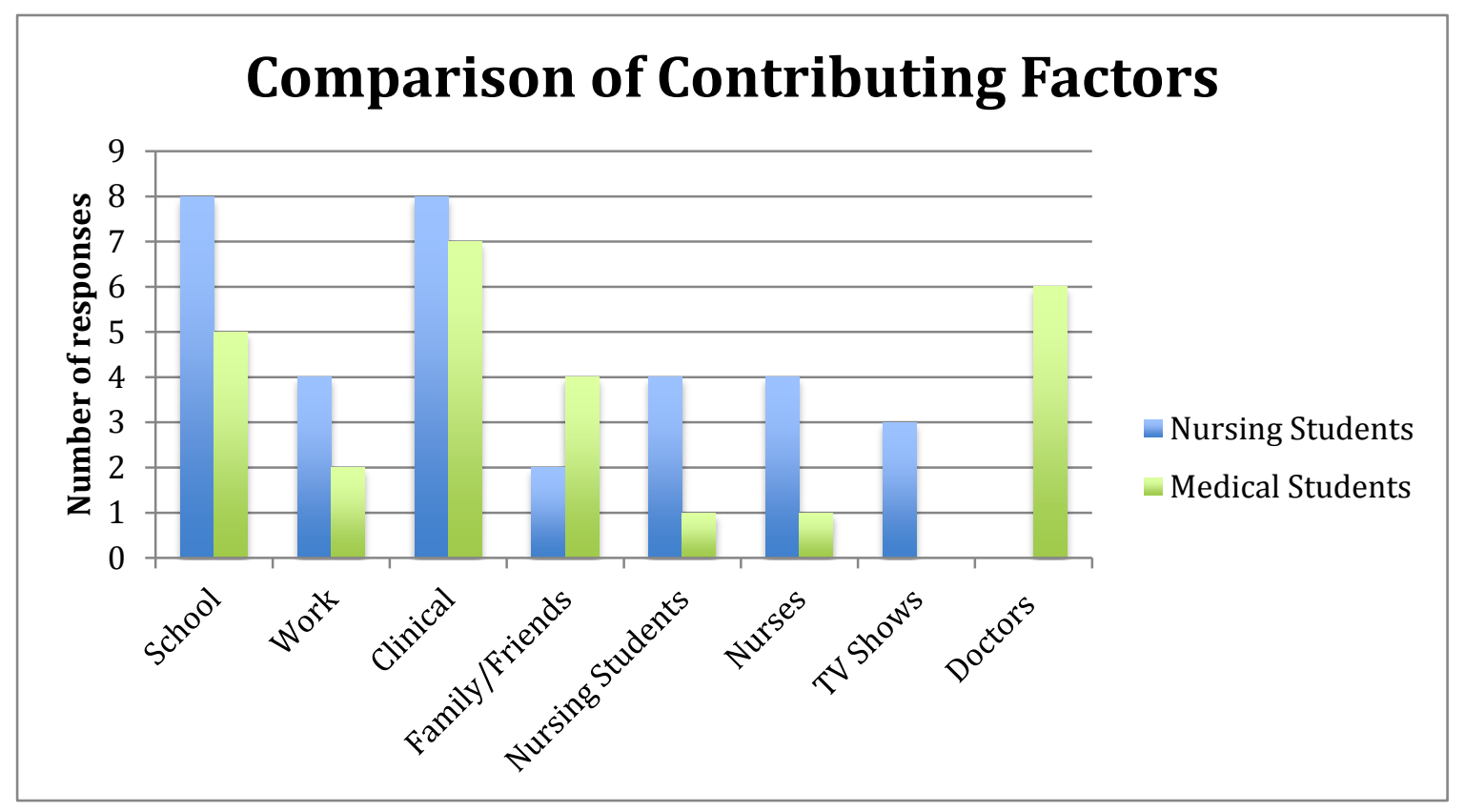

Figure 3. Nursing and medical students contributing factors to perceptions compared from 16 research interviews

\section{Implications for Nursing Practice}

Nurses are impacted by the nurse- physician relationship every day as they seek to provide quality patient care at the bedside. Research in understanding the nurse- physician relationship helped staff a better understanding of what biases are affecting this professional relationship. This study generated enough information to implement helpful tools to allow nurses to be better educated on their own personal biases against physicians and the ways they can collaborate and communicate more effectively. The result of this study shows the impact of the nurse- 
physician relationship and its contributing factors have on the healthcare professional's view of the nursephysician relationship.

\section{Limitations}

Comparable to all qualitative studies, this research was limited by having subjective data. The information was based on personal experiences and then interpreted by inexperienced researchers. Despite this limitation, information regarding this subject was still important to assess the perceptions of the nurse- physician relationship. Another limitation was the small sample size of only 16 participants. The results from the small sample size cannot be transferred to other medical or nursing students outside of the Midwest.

One other limitation was the time frame in which the interviews took place. The researchers anticipated the interviews to take 45 minutes to an hour. However, the interviews only took between 20 to 30 minutes on average. Another limitation was the skill of the interviewer and the analysis of the information received. These qualitative methods are dependent on the personal exchanges with respondents, and variables that affected the results included the attitude, language, and disposition used by the interviewer. The experiences of the researchers also influenced how well the data was summarized into themes.

\section{Recommendations}

Teamwork and collaboration is a topic of concern in the relationship between nurses and physicians. Our recommendation is that the nursing programs and medical schools would educate on the importance of working together as a team in order to improve patient care. A major challenge in working together collaboratively in healthcare is communication and in order to remedy this, we recommend that nursing and medical students are encouraged to seek out opportunities to communicate with the opposite role in the clinical setting as much as possible to gain experiences. Further studies could be done on a national level to see if the same findings apply to the nurse- physician relationship in other states. A national study would aid in validity of results and determine whether further education would be beneficial in nursing and medical schools across the nation.

\section{Conclusion}

The research explored themes provided by nursing students and medical students in open-ended interviews. Compared to previous literature, this research study found that there are barriers in the nurse-physician and medical student-physician relationships. Positive contributing factors based on this study for nursing students-physician relationship include being respectful toward each other, value each other, appreciate each other, and act professional. Negative contributing factors based on this study for nursing students-physician relationship included inadvertently disrespectful, acting superior, condescending, and stereotypical behavior. On the other hand, positive contributing factors based on this study for medical students-physician relationship included being respectful toward each other and appreciation of experience. Stereotypical, disrespectful, and sexist attitudes were prominent negative characteristics noted among medical students-physician relationships. This research informs nurses and physicians of the barriers in their professional relationships and the ways they can identify their own personal biases.

\section{References}

Brown, J., Hochstetler, G. A., Rode, S. A., Abraham, S. P., \& Gillum, D. R. (2018). The lived experience of first-year nurses at work. The Health Care Manager, 37(4), 281-289. https://doi.org/10.1097/HCM.0000000000000228

Collette, A. E., Wann, K., Nevin, M. L., Rique, K., Tarrant, G., Hickey, L. A., ...Thomason, T. (2017). An exploration of nurse-physician perceptions of collaborative behavior. Journal of Interprofessional Care, 31(4), 470-479. https://doi.org/10.1080/13561820.2017.1301411

Guidelines for Establishing Joint or Collaborative Practice in Hospitals. (1974). A demonstration project directed by the National Joint Practice Commission. Chicago, IL: Nealy Printing Co.

House, S., \& Havens, D. (2017). Nurses' and physicians' perceptions of nurse-physician collaboration. Journal of Nursing Administration, 47(3), 165-171. https://doi.org/10.1097/NNA.0000000000000460

Krueger, L., Ernstmeyer, K., \& Kirking, E. (2017). Impact of interprofessional simulation on nursing student's attitudes toward teamwork and collaboration. Journal of Nursing Education, 56(6), 321-327. https://doi.org/10.3928/01484834-20170518-02

Lancaster, G., Kolakowsky-Hayner, S., Kovacich, J., \& Greer-Williams, N. (2015). Interdisciplinary communication and collaboration among physicians, nurses, and unlicensed assistive personnel. Journal of Nursing Scholarship, 47(3), 275-284. https://doi.org/10.1111/jnu.12130 
Schaik, S. M., O’Sullivan, P. S., Eva, K. W., Irby, D. M., \& Regehr, G. (2016) Does source matter? Nurses' and physicians' perceptions of interprofessional feedback. Medical Education, 50(2), 181-188. https://doi.org/10.1111/medu.12850

Schmidt, N., \& Brown, J. (2019). Evidence-based practice for nurses: Appraisal and application of research (4th ed.). Sudbury, MA: Jones \& Bartlett Learning.

Speziale, H. J., \& Carpenter, D. R. (2007). Qualitative research in nursing: Advancing the humanistic imperative (4th ed.). Philadelphia, PA: Lippincott, Williams, and Wilkins.

Stein, L. I. (1967). The doctor-nurse game. Archives of General Psychiatry, 16(6), 699-703. https://doi.org/10.1001/archpsyc.1967.01730240055009

Tajfel, H. (1982). Social psychology of intergroup relations. Annual Review of Psychology, 33(1), 1-39. http://dx.doi.org/10.1146/annurev.ps.33.020182.000245

Tang, C., Chan, S., Zhou, W., \& Liaw, S. (2013). Collaboration between hospital physicians and nurses: An integrated literature review. International Nursing Review, 60(3), 291-302. https://doi.org/10.1111/inr.12034

The Joint Commission. (2008). The Joint Commission on Accreditation of Healthcare Organizations. Sentinel event alert: Behaviors that undermine a culture of safety. Retrieved from https://www.jointcommission.org/assets/1/18/SEA_40.PDF

Thomson, K., Outram, S., Gilligan, C., \& Levett-Jones, T. (2015). Interprofessional experiences of recent healthcare graduates: A social psychology perspective on the barriers to effective communication, teamwork, and patient-centered care. Journal of Interprofessional Care, 29(6), 634-640. https://doi.org/10.3109/13561820.2015.1040873

Vila, L. L. (2016). Physician perceptions of Magnet nurses and Magnet designation. Journal of Nursing Care Quality, 31(4), 380-386. https://doi.org/10.1097/NCQ.0000000000000195

\section{Copyrights}

Copyright for this article is retained by the author(s), with first publication rights granted to the journal.

This is an open-access article distributed under the terms and conditions of the Creative Commons Attribution license (http://creativecommons.org/licenses/by/4.0/). 